\title{
Características oclusales de los niños de 5 años del municipio de andes (Antioquia, Colombia)
}

\author{
Occlusal characteristics in children 5-year-old of Andes
} municipality (Antioquia, Colombia)

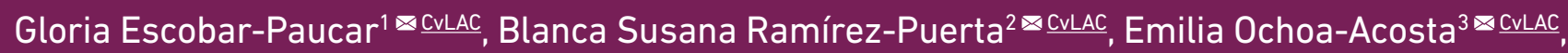
Ángela María Franco-Cortes ${ }^{4} \bowtie \underline{C V L A C}$

1. Odontopediatra. Magíster en Salud Pública. Profesora titular Universidad de Antioquia. Medellín, Colombia.

2. Odontóloga. Especialista en Gerencia de la Salud Pública. Magíster en epidemiología. Profesora titular Universidad de Antioquia.Medellín,

Colombia.

3. Magíster en educación y desarrollo humano. Profesora ocasional Universidad de Antioquia. Profesora Universidad Cooperativa de Colombia.

Medellín, Colombia.

4. Odontóloga. Magíster en epidemiología .PhD ciencias sociales, niñez y juventud. Profesora titular Universidad de Antioquia. Medellín, Colombia.

Fecha correspondencia:

Recibido: septiembre de 2016.

Aceptado: noviembre de 2016.

\section{Forma de citar:}

Escobar-Paucar G, Ramírez-Puerta BS, Ochoa-Acosta E, Franco-Cortes AM. Características oclusales de los niños de 5 años del municipio de andes (Antioquia, Colombia). Rev. CES Odont 2016; 29(2): 33-39.

Open access

(C) Derecho de autor

Licencia creative commons

Ética de publicaciones

Revisión por pares

Gestión por Open Journal System

ISSN 0120-971X

e-ISSN 2215-9185

Comparte

\section{Resumen}

Introducción: Las alteraciones oclusales son un problema frecuente en la dentición primaria, su identificación desde edades tempranas permitirá reconocer variaciones en las características normales, que pueden influir en el desarrollo de la oclusión permanente. Objetivo: Establecer las características oclusales en dentición primaria, de los niños y niñas de 5 años de la zona urbana y rural del municipio de Andes (Colombia). Materiales y métodos: Se realizó una valoración clínica de 125 niños mediante inspección visual de la relación entre los componentes dentarios. Se calculó la prevalencia para cada una de las alteraciones oclusales y se estimaron además la frecuencia de alteraciones combinadas. Resultados: Se encontró relación canina clase I en el $84 \%$ y clase II con un $12 \%$. La sobremordida horizontal y la vertical fueron normales en el $60,8 \%$ y en el $58,4 \%$ respectivamente y el 6,4\% tenían mordida cruzada posterior. No se encontró ninguna alteración oclusal en el $48,8 \%$ de los niños; mientras que en el 3,2\% las 4 características oclusales evaluadas estaban alteradas. No hubo diferencias estadísticamente significativas en las características oclusales según sexo. Conclusión: La presencia de alteraciones en la oclusión, detectadas en mayor proporción que el promedio nacional, requiere implementar en el municipio acciones para su detección y tratamiento oportuno que contribuyan a un adecuado crecimiento y desarrollo craneofacial.

Palabras clave: Salud bucal, dentición primaria, niños, oclusión dental

\section{Abstract}

Introduction: The occlusal alterations are a frequent problem in the primary dentition, their identification from early age, allow to recognize variations in the normal characteristics, which can influence the development of permanent occlusion. Objective: To establish the occlusal characteristics in primary dentition, in children 5-year-old of Andes municipality, Colombia. Materials and methods: One hundred twenty five children were 
evaluated by a clinical visual inspection of the relationship between the dental components. Prevalence for each occlusal characteristic was calculated and the combined frequencies of alterations were also estimated. Results: Class I canine relationship in $84 \%$ and Class II in 12\% were found. The horizontal and vertical overbite were normal in $60.8 \%$ and $58.4 \%$ respectively, and $6.4 \%$ had posterior cross bite. None occlusal alteration was found in $48.8 \%$ of children; while $3.2 \%$ had alteration in the four occlusal characteristics evaluated. There were no statistically significant differences in occlusal characteristics by sex. Conclusion: The presence of occlusal alterations, in a greater proportion than the national average, requires implementing in the municipality actions for its detection and treatment that contribute to adequate craniofacial growth and development.

Keywords: Oral health, primary dentition, children, occlusal characteristics, dental occlusion.

\section{Introducción}

El complejo craneofacial es un sistema que apoya e interactúa con varias funciones vitales. Algunas alteraciones oclusales que involucran la relación de los maxilares en los planos vertical, sagital y transversal, influyen el desarrollo craneofacial del individuo desde temprana edad produciendo alteraciones en la función masticatoria y respiratoria cuando no se tratan a tiempo. Igualmente las irregularidades o malposiciones dentarias son consideradas factores retentivos que favorecen la acumulación o impactación de placa dentobacteriana y biopelícula dental, favoreciendo el desarrollo de caries dental, porque se convierten en zonas de difícil acceso con el cepillo dental (1- $\underline{1})$.

Si bien hay menos variabilidad en las relaciones oclusales en la dentición primaria que en la dentición permanente (4), la presencia de ciertas características puede implicar el desarrollo de maloclusiones más complejas o anomalías esqueléticas en la dentición permanente; en casos como la mordida cruzada posterior, se recomienda realizar tratamiento temprano para prevenir un posicionamiento asimétrico de los cóndilos, y con ello asimetrías en el crecimiento mandibular (므). Identificar la presencia de alteraciones oclusales combinadas en dentición primaria, permitiría actuar de manera oportuna y reducir los costos de tratamiento de maloclusiones más complejas, con beneficios a mediano y largo plazo en el logro de objetivos de armonía oclusal, función y estética dentofacial ()ㅡ).

Pocos estudios en Colombia reportan datos sobre la condición de la oclusión en dentición primaria (). aunque esta presenta variaciones importantes entre grupos poblacionales (8) y la mayoría se refieren a usuarios que acuden a servicios odontológicos $(\underline{9}, \underline{10})$.

El III Estudio Nacional de Salud Bucal - ENSAB III realizado en 1998, identificó algunas condiciones (11), pero no evalúo la oclusión en la dentición primaria. El último estudio nacional de salud bucal realizado en el país en 2014 -ENSAB IV- aportó información para conocer más acerca del problema en niños y niñas de 5 años en Colombia (12), pero los resultados se presentan para la población general y no permiten hacer análisis por regiones.

La identificación de alteraciones oclusales desde edades tempranas en un territorio específico permitirá reconocer condiciones que pueden influir el desarrollo de la oclusión permanente, e identificar necesidades de tratamiento presentes y futuras 
para la organización de los servicios. El objetivo de este estudio fue establecer las características oclusales de dentición primaria de los niños y niñas de 5 años del municipio de Andes (Colombia).

\section{Materiales y métodos}

Los datos fueron recolectados en un estudio descriptivo transversal realizado en el año 2014 para la obtención de indicadores de salud en niños y niñas de 0 a 5 años del municipio de Andes, como línea de base en el marco del proyecto Alianza por un Futuro Libre de Caries. La muestra se calculó con un nivel de confianza (Z) del $95 \%$, un error de muestreo (e) del $2 \%$, y se tuvo en cuenta el factor de corrección de población finita. El tamaño de la muestra para todas las edades fue 717 niños y niñas, y se evaluaron finalmente 642; de los cuales 125 tenían cinco años de edad, a quienes se les realizó evaluación de las características oclusales. El protocolo del estudio fue aprobado por el Comité de Ética de la Facultad de Odontología de la Universidad de Antioquia (acta № 222013 del 15 de noviembre de 2014). Se contó con el consentimiento informado del adulto responsable y el asentimiento del menor para la evaluación clínica.

Las características oclusales de los niños en dentición primaria, se evaluaron mediante inspección visual de la relación entre los componentes dentarios con base en el índice de Foster y Hamilton (13). El examen se realizó en una unidad portátil, utilizando fotóforo, espejo intraoral y sonda UNC-15. Se evalúo relación canina (clase I, II o III), sobremordida horizontal (normal, aumentada, borde a borde, cruzada anterior), sobremordida vertical (normal, disminuida, abierta, profunda), y se registró la presencia o ausencia de mordida cruzada posterior.

La evaluación clínica fue realizada por dos odontólogos que participaron en la estandarización del ENSAB IV, y el registro de los hallazgos del examen se hizo con base en criterios diagnósticos utilizados en dicho estudio, que retomó la propuesta de evaluación de características oclusales del estudio de Brasil 2010 (14).

Para el análisis de la información, se elaboró una base de datos en Microsoft Excel ${ }^{\circledR}$ y se exportó al programa estadístico IBM SPSS Statistics $19.0^{\circledR}$ para el cálculo de la prevalencias de las diferentes categorías de alteraciones oclusales. Se exploraron diferencias según sexo y zona en el programa Epidat 3.1, mediante la prueba Chi-cuadrado de Pearson.

\section{Resultados}

Se analizó la oclusión en 125 niños y niñas de 5 años, 63 (50,4\%) de sexo femenino y $62(49,6 \%)$ masculino. El 73,6\% de ellos residentes en la zona rural del municipio.

En la tabla 1 se presentan la distribución porcentual de las características oclusales evaluadas, siendo las más comunes la relación canina clase I en el $84 \%$, la sobremordida horizontal y vertical normales en un $60,8 \%$ y $58,4 \%$ respectivamente, y ausencia de mordida cruzada posterior en el $92 \%$ de los niños evaluados.

No se encontraron diferencias estadísticamente significativas en las características oclusales de los niños y niñas de 5 años según sexo ni por zona de residencia (Tabla 2).

Cuando se analizó la frecuencia de alteraciones oclusales combinadas, se encontró que el $48,8 \%$ de los niños/as presentaron las cuatro características evaluadas (rela- 
Tabla 1. Distribución de las características oclusales de los niños y niñas de 5 años. Andes (Antioquia), 2014

\begin{tabular}{|c|c|c|c|c|c|c|c|c|}
\hline \multirow{2}{*}{$\begin{array}{c}\text { Características } \\
\text { oclusales }\end{array}$} & \multirow{2}{*}{ Categorías } & \multicolumn{2}{|c|}{ Niñas } & \multicolumn{2}{|c|}{ Niños } & \multicolumn{2}{|c|}{ Total } & \multirow{2}{*}{$\begin{array}{c}\text { Valor } \\
p^{*}\end{array}$} \\
\hline & & $n$ & $\%$ & $n$ & $\%$ & $n$ & $\%$ & \\
\hline \multirow{4}{*}{$\begin{array}{l}\text { Relación } \\
\text { canina }\end{array}$} & Clase I & 54 & 85,7 & 51 & 82,3 & 105 & 84 & \multirow{4}{*}{0,479} \\
\hline & Clase II & 8 & 12,7 & 7 & 11,3 & 15 & 12 & \\
\hline & Clase III & 1 & 1,6 & 2 & 3,2 & 3 & 2,4 & \\
\hline & Sin información ${ }^{\dagger}$ & 0 & 0 & 2 & 3,2 & 2 & 1,6 & \\
\hline \multirow{5}{*}{$\begin{array}{l}\text { Sobremordida } \\
\text { horizontal }\end{array}$} & Normal & 43 & 68,3 & 33 & 53,2 & 76 & 60,8 & \multirow{5}{*}{0,261} \\
\hline & Aumentada & 7 & 11,1 & 6 & 9,7 & 13 & 10,4 & \\
\hline & Borde a borde & 5 & 7,9 & 8 & 12,9 & 13 & 10,4 & \\
\hline & Cruzada anterior & 0 & 0 & 2 & 3,2 & 2 & 1,6 & \\
\hline & Sin información ${ }^{\dagger}$ & 8 & 12,7 & 13 & 21 & 21 & 16,8 & \\
\hline \multirow{5}{*}{$\begin{array}{l}\text { Sobremordida } \\
\text { vertical }\end{array}$} & Normal & 40 & 63,5 & 33 & 53,2 & 73 & 58,4 & \multirow{5}{*}{0,681} \\
\hline & Disminuida & 12 & 19 & 12 & 19,4 & 24 & 19,2 & \\
\hline & Abierta & 2 & 3,2 & 2 & 3,2 & 4 & 3,2 & \\
\hline & Profunda & 1 & 1,6 & 1 & 1,6 & 2 & 1,6 & \\
\hline & Sin información ${ }^{\dagger}$ & 8 & 12,7 & 14 & 22,6 & 22 & 17,6 & \\
\hline \multirow{3}{*}{$\begin{array}{l}\text { Mordida cruzada } \\
\text { posterior }\end{array}$} & Ausencia & 58 & 92,1 & 57 & 91,9 & 115 & 92 & \multirow{3}{*}{0,286} \\
\hline & Presencia & 5 & 7,9 & 3 & 4,8 & 8 & 6,4 & \\
\hline & Sin información ${ }^{\dagger}$ & 0 & 0 & 2 & 3,2 & 2 & 1,6 & \\
\hline Total & & 63 & 50,4 & 62 & 49,6 & 125 & 100 & \\
\hline
\end{tabular}

${ }^{*}$ Chi Cuadrado de Pearson

†Corresponde a los niños en los que no fue posible el registro debido a la ausencia de piezas dentales que permitieran la evaluación de la característica.

Tabla 2. Distribución de las características oclusales de los niños y niñas de 5 años, según zona de residencia. Andes (Antioquia), 2014

\begin{tabular}{|c|c|c|c|c|c|c|c|c|}
\hline \multirow{2}{*}{$\begin{array}{c}\text { Características } \\
\text { oclusales }\end{array}$} & \multirow{2}{*}{ Categorías } & \multicolumn{2}{|c|}{ Zona rural } & \multicolumn{2}{|c|}{ Zona urbana } & \multicolumn{2}{|c|}{ Total } & \multirow{2}{*}{ Valor $\boldsymbol{p}^{*}$} \\
\hline & & $n$ & $\%$ & $n$ & $\%$ & $n$ & $\%$ & \\
\hline \multirow{4}{*}{ Relación canina } & Clase I & 76 & 82,6 & 29 & 87,9 & 105 & 84 & \multirow{4}{*}{0,202} \\
\hline & Clase II & 13 & 14,1 & 2 & 6,1 & 15 & 12 & \\
\hline & Clase III & 1 & 1,1 & 2 & 6,1 & 3 & 2,4 & \\
\hline & Sin información ${ }^{\dagger}$ & 2 & 2,2 & 0 & 0,0 & 2 & 1,6 & \\
\hline \multirow{5}{*}{$\begin{array}{l}\text { Sobremordida } \\
\text { horizontal }\end{array}$} & Normal & 54 & 58,7 & 22 & 66,7 & 76 & 60,8 & \multirow{5}{*}{0,380} \\
\hline & Aumentada & 8 & 8,7 & 5 & 15,2 & 13 & 10,4 & \\
\hline & Borde a borde & 11 & 12,0 & 2 & 6,1 & 13 & 10,4 & \\
\hline & Cruzada anterior & 1 & 1,1 & 1 & 3,0 & 2 & 1,6 & \\
\hline & Sin información ${ }^{\dagger}$ & 18 & 19,6 & 3 & 9,1 & 21 & 16,8 & \\
\hline \multirow{5}{*}{$\begin{array}{l}\text { Sobremordida } \\
\text { vertical }\end{array}$} & Normal & 51 & 55,4 & 22 & 66,7 & 73 & 58,4 & \multirow{5}{*}{0,090} \\
\hline & Disminuida & 20 & 21,7 & 4 & 12,1 & 24 & 19,2 & \\
\hline & Abierta & 3 & 3,3 & 1 & 3,0 & 4 & 3,2 & \\
\hline & Profunda $>100 \% ?$ & 0 & 0,0 & 2 & 6,1 & 2 & 1,6 & \\
\hline & Sin información ${ }^{\dagger}$ & 18 & 19,6 & 4 & 12,1 & 22 & 17,6 & \\
\hline \multirow{3}{*}{$\begin{array}{l}\text { Mordida cruzada } \\
\text { posterior }\end{array}$} & Ausencia & 84 & 91,3 & 31 & 93,9 & 115 & 92 & \multirow{3}{*}{0,689} \\
\hline & Presencia & 6 & 6,5 & 2 & 6,1 & 8 & 6,4 & \\
\hline & Sin información ${ }^{\dagger}$ & 2 & 2,2 & 0 & 0,0 & 2 & 1,6 & \\
\hline Total & & 92 & 73,6 & 33 & 26,4 & 125 & 100 & \\
\hline
\end{tabular}

${ }^{*}$ Chi Cuadrado de Pearson

${ }^{\dagger}$ Corresponde a los niños en los que no fue posible el registro debido a la ausencia de piezas dentales que permitieran la evaluación de la característica. 
ción canina, sobremordida horizontal, sobremordida vertical y mordida cruzada posterior) dentro de los parámetros normales; mientras que el 37,6\% presentó alteración de 2 o más características oclusales, y un 3,2\% presentó alteración en todas, (Figura 1).

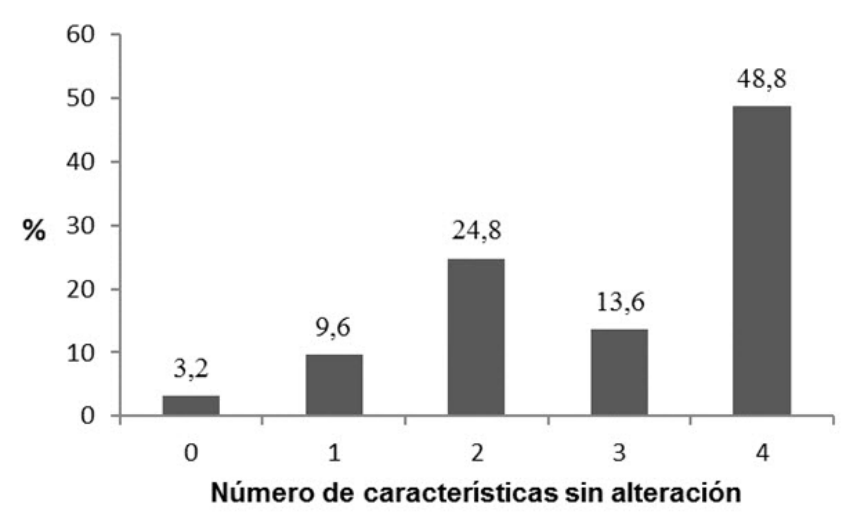

Figura 1. Distribución de los niños de 5 años según número de características oclusales sin alteración. Andes (Antioquia), 2014

\section{Discusión}

Si bien el hallazgo más común en los niños y niñas de Andes correspondió a aquellas características oclusales consideradas normales, se encuentran diferencias en comparación con los datos de los estudios nacionales de Colombia y Brasil, los cuales emplean parámetros similares. La relación canina clase I se encontró en el $84 \%$ en los niños evaluados, mientras que el dato nacional para Colombia fue del 95,5\% y $77,1 \%$ para Brasil. La sobremordida horizontal está presente en el $60,8 \%$ de los niños, en comparación con el $71 \%$ en el estudio nacional y porcentajes que varían entre $60,8 \%$ a $71,2 \%$ entre regiones en Brasil. Por su parte, la sobremordida vertical normal está presente en un 58,4\% de los niños, en comparación con el 83,6\% nacional $(\underline{12}, \underline{15})$. Todas las características muestran un comportamiento similar en ambos sexos, como es de esperar a estas edades.

El $92 \%$ de los niños evaluados no presentaron mordida cruzada posterior. El $8 \%$ restante, cifra similar al 7,25 reportado en un estudio poblacional en Bogotá (7), requerirían un tratamiento lo más pronto posible para corregir esta condición de acuerdo con las recomendaciones vigentes, en tanto las mordidas cruzadas posteriores tienen implicaciones para el desarrollo oclusal y craneofacial armónico $(\underline{6}, \underline{16})$. La prevalencia de esta alteración oclusal es ligeramente inferior al $10.1 \%$ reportado en Brasil. Por su parte, la mordida cruzada anterior cuyo tratamiento temprano también está indicado, presentó una prevalencia de 3,7\%, igual al estudio nacional y similar a Brasil $(3,0 \%)(\underline{12}, \underline{15})$.

Una limitación para realizar comparaciones con otros estudios realizados en el país $(\underline{9}, \underline{10})$, radica en que en este estudio no se tuvo en cuenta la relación molar, una característica de gran relevancia clínica, pero que en otros estudios poblacionales tampoco se ha considerado, tomando como referencia la relación canina para evaluar la armonía en el plano sagital.

En cuanto a la frecuencia de alteraciones oclusales combinadas, también se encontró un porcentaje menor de normalidad en Andes: 48,8\% presentaron las cuatro características evaluadas en los rangos normales, en comparación con el 53,1 \% en la población nacional. 
El 37,6\% presentó alteración de 2 o más características oclusales, en comparación con un $18 \%$ en el estudio nacional, y un 3,2\% presentó alteración en todas las características. Ello refleja mayores necesidades de tratamiento en esta población, lo cual contrasta con la menor oferta de servicios en comparación con los grandes centros urbanos.

\section{Conclusión}

Si bien enfermedades como la caries dental constituyen la mayor carga de enfermedad bucal en niños de cinco años en esta y en otras poblaciones $(\underline{17}, 18)$, es importante tomar conciencia de la presencia de alteraciones en la oclusión, para detectarlas a tiempo e implementar intervenciones tempranas que contribuyan a un adecuado crecimiento y desarrollo craneofacial y por ende a la función y el bienestar.

\section{Agradecimientos}

A la Universidad de Antioquia, el Municipio de Andes y la empresa Colgate Palmolive, por los aportes para la ejecución del Proyecto Alianza por un futuro libre de caries, capítulo Colombia. Código centro de investigación Facultad de Odontología número 18-2013; del cual se derivó este artículo.

\section{Referencias bibliográficas}

1. Uribe G. Ortodoncia Teoría y Clínica. Fundamentos de odontología. 2da edición. Medellín: CIB; 2010.1312 p.

2. Cárdenas D. Odontología Pediátrica Fundamentos de odontología. 4 a edición. Medellín: CIB; 2009. 560 p.

3. González MC, Valbuena LF, Zarta OL, Martignon S, Arenas M, Leaño M, Ekstrand KR, Christiansen Ch. Guías de práctica clínica basadas en la evidencia: Caries Dental. Proyecto ISS-ACFO. Manizales: Editorial gráficas JES; 1998.

4. Saadia AM. Development of occlusion and oral funtion in children. The J Pedod. 1981;5(2):154-172. https://www.ncbi.nlm.nih.gov/pubmed/6942147

5. Kanellis MJ. Tratamiento ortodóntico en la dentición primaria. En: Bishara SE, ed. Ortodoncia. México: MCGraw-Hill Interamericana; 2003: pp 268-277.

6. American Academy of Pediatric Dentistry. Guideline on management of the developing dentition and occlusion in pediatric dentistry. Reference manual. Clinical practice guidelines 2015 - 2016. Ped Dent 2015;37 (6):253-265. http://www. aapd.org/media/policies guidelines/g developdentition.pdf

7. Thilander B, Pena L, Infante C, Parada SS, de Mayorga C. Prevalence of malocclusion and orthodontic treatment need in child and adolescents in Bogotá, Colombia. An epidemiological study related to different stages of dental development. The European Journal of Orthodontics 2001;23(2):153-167. https://academic.oup. com/ejo/article/23/2/153/440815/Prevalence-of-malocclusion-and-orthodontic

8. Moslemi M, Nadalizadeh S, Sarsanghizadeh S, Sadrabad S, Shadkar S, Shadkar MS. Evaluation of Dental Occlusion in 3-5 year-Old Children. IJMRI 2015;1(1):4853. 
9. Botero P, González S, Correa D. Necesidad de tratamiento ortodóntico en niños que consultan a la Universidad Cooperativa de Colombia. Revista Nacional de Odontología 2009;5(9):9-14. http://www.imbiomed.com/1/1/articulos.php?method=showDetail\&id articulo=65036\&id seccion=3840\&id ejemplar=6536\&id revista $=231$

10. Botero P, Vélez N, Cuesta DP, Gómez E, González PA, Cossio M, Santos E. Perfil epidemiológico de oclusión dental en niños que consultan a la Universidad Cooperativa de Colombia. Rev CES Odont 2009;22(1)9-13. http://revistas.ces.edu.co/ index.php/odontologia/article/view/502

11. Colombia. Ministerio de Salud. III Estudio Nacional de Salud Bucal. Serie Documentos Técnicos. Tomo VII. Bogotá, 1999. http://www.visitaodontologica.co/ ARCHIVOS/ARCHIVOS-NORMAS/Salud\%20Publica_P_y_P/II ESTUDIO NACIONAL SALUD BUCAL.pdf

12. Colombia. Ministerio de Salud y Protección Social. IV Estudio Nacional de Salud Bucal. ENSAB IV: Para saber cómo estamos y saber qué hacemos. Bogotá: Ministerio de Salud y Protección Social 2013-2014. Disponible en:https://www. minsalud.gov.co/sites/rid/Lists/BibliotecaDigital/RIDE/VS/PP/ENSAB-IV-Situacion-Bucal-Actual.pdf. Consultado junio 7 de 2016.

13. Foster TD, Hamilton MC. Occlusion in the primary dentition: study of children at $21 / 2$ to 3 years of age. Br Dent J 1969;126(2):76-79. https://www.ncbi.nlm.nih. gov/pubmed/5253447

14. Brasil. Ministério da Saúde Secretaria de Atenção à Saúde Departamento de Atenção Básica Coordenação Nacional de Saúde Bucal. Pesquisa Nacional de Saúde Bucal. Projeto técnico. Brasília, DF Setembro de 2009. Disponible en http://dab.saude.gov.br/CNSB/sbbrasil/arquivos/Projeto SB2010 VersaoFinal. pdf. Consultado mayo 14 de 2016.

15. Brasil. Ministério da Saúde. Secretaria de Atenção à Saúde. Secretaria de Vigilância em Saúde. SB Brasil 2010: Pesquisa Nacional de Saúde Bucal: resultados principais, Brasília, 2012. Disponible en: http://dab.saude.gov.br/CNSB/sbbrasil/ arquivos/projeto sb2010 relatorio final.pdf. Consultado mayo 14 de 2016.

16. Pinto AS, Buschang PH, Throckmorton GS, Chen P. Morphological and positional asymmetries of young children with functional unilateral posterior crossbite. Am J Orthod Dentofacial Orthop 2001;120(5):513-520. https://www.ncbi.nlm.nih. gov/pubmed/11709670

17. Ramírez-Puerta BS, Escobar-Paucar G, Fanco-Cortés AM, Ochoa- Acosta EM, Otálvaro-Castro GJ, Agudelo-Suárez AA. Caries Dental en niños de 0-5 años del Municipio de Andes (Colombia), usando El Sistema Internacional de Detección y Evaluación de Caries - ICDAS. Aprobado para publicación en la Revista Facultad Nacional de Salud Pública. https://aprendeenlinea.udea.edu.co/revistas/index. $\mathrm{php/fnsp/article/view/26344}$

18. Marcenes W, Kassebaum NJ, Bernabé E, Flaxman A, Naghavi M, Lopez A, Murray CJ. Global Burden of Oral Conditions in 1990-2010: A Systematic Analysis. J Dent Res 2013;92(7):592-597. https://www.ncbi.nlm.nih.gov/pubmed/23720570 